\title{
A new generated distribution to analyze a practical engineer- ing problem and applications
}

\author{
Enayat M. Abd Elrazik ${ }^{\mathrm{a}}$, Mahmoud M. Mansour ${ }^{\mathrm{b}, *}$ \\ a Department of MIS, Yanbu, Taibah University, Saudi Arabia. \\ ${ }^{b}$ Department of Statistics, Mathematics and Insurance, Benha University, Egypt.
}

\begin{abstract}
There are many systems that can handle a mix of series-parallel or parallel-series systems. Here, a new three-parameter distribution motivated mainly by dealing with series-parallel or parallel-series systems is introduced. Moments, conditional moments, mean deviations, moment generating function, quantile, Lorenz, and Bonferroni curves of the new distribution including are presented. Entropy measures are given and estimation of its parameters is studied. Two real data applications are described to show its superior performance versus some known lifetime models.
\end{abstract}

Keywords: Lindley distribution, geometric distribution, maximum likelihood estimation, truncated Poisson distribution. 2010 MSC: 60E05, 62E10, 62E15.

(C)2019 All rights reserved.

\section{Introduction}

This research aims at introducing a new three-parameter lifetime distribution with physical motivations. As explained below, the proposed distribution gives preferred fits over a large number of the known lifetime distributions, including those with four parameters.

Our second motivation is based on a practical situation, where a factory has two parallel production lines. The two lines are assembled together before going to the market. However, the production manager is interested in analyzing the characteristics of each production line separately. The problem here is that one line consists of machines in sequence (connected in series) with a replicated of each individual machine, while the other line is a collection of machines connected in tandem as subsystems and the subsystems are connected in parallel.

In this paper we provide a new general distribution for handle with series parallel system, and we use the method introduced by Nadarajah et al. [8] to analyze parallel series system.

The proposed distributions that can handle with series-parallel or parallel-series configuration, i.e., Mini $\operatorname{Max} X_{i}$ or Max Mini $X_{i}$, where $X_{i}$ denote the failure time of the $i$ th system. Then, the cumulative distribution function of $X$, say $G(x)$, can be derived as follows.

\footnotetext{
*Corresponding author

Email addresses: ekhalilabdelgawad@taibahu.edu.sa (Enayat M. Abd Elrazik), mmmansour@taibahu.edu.sa (Mahmoud M. Mansour)
}

doi: $10.22436 /$ jnsa.012.07.05

Received: 2018-07-05 Revised: 2018-10-05 Accepted: 2019-03-09 


\subsection{Mini Max Distribution}

Nadarajah et al. [8] introduced a two-parameter distribution which represent a general model by taking the probability density function of the cumulative distribution function of failure times to be given by $f($.$) and F($.$) , respectively. Its cdf is given by$

$$
G(x)=\frac{\exp (-\lambda+\lambda F(x))-e^{-\lambda}}{1-e^{-\lambda}-\pi+\pi \exp (-\lambda+\lambda F(x))},
$$

for $x>0, \lambda>0$ and $0<\pi<1$. The corresponding probability density function is,

$$
g(x)=\frac{\lambda(1-\pi)\left(1-e^{-\lambda}\right) f(x) \exp (-\lambda+\lambda F(x))}{\left\{1-e^{-\lambda}-\pi+\pi \exp (-\lambda+\lambda F(x))\right\}^{2}} .
$$

Many studies that are interested for analyzing lifetime data have used Lindely to analyze lifetime data. So, we took failure times to follow the Lindley distribution. A random variable $X$ is said to have a Lindley distribution with parameter $\theta$ if its probability density is defined as,

$$
f(x)=\frac{\theta^{2}}{\theta+1}(1+x) e^{-\theta x}, x>0, \theta>0 .
$$

The corresponding cumulative distribution function (cdf) is:

$$
F(x)=1-\frac{\theta+1+\theta x}{\theta+1} e^{-\theta x}, x>0, \theta>0
$$

Assume that the failure times of the units for the $i^{\text {th }}$ system, say $Z_{i, 1}, Z_{i, 2}, \ldots, Z_{i, M}$, are independent and identical Lindley random variables with the scale Parameter $\theta$. Let $Y_{i}$ denote the failure time of the $i$ th system. Let $X$ denote the time to failure of the first of the $N$ functioning systems. We can write $X=\min \left(Y_{1}, Y_{2}, \ldots, Y_{n}\right)$. By using (1.1), (1.2), and (1.3), the conditional cumulative distribution function of $X$ given $N$ is,

$$
G(x)=\frac{\exp \left(-\lambda\left(\frac{\theta+1+\theta x}{1+\theta}\right) e^{-\theta x}\right)-e^{-\lambda}}{1-e^{-\lambda}-\pi\left[1-\exp \left(-\lambda\left(\frac{\theta+1+\theta x}{1+\theta}\right) e^{-\theta x}\right)\right]},
$$

for $x>0, \theta>0, \lambda>0$, and $0<\pi<1$. The corresponding probability density function is,

$$
g(x)=\frac{\lambda \theta^{2}(1-\pi)\left(1-e^{-\lambda}\right)(1+x) \exp \left(-\theta x-\lambda\left(\frac{\theta+1+\theta x}{1+\theta}\right) e^{-\theta x}\right)}{(1+\theta)\left\{1-e^{-\lambda}-\pi\left[1-\exp \left(-\lambda\left(\frac{\theta+1+\theta x}{1+\theta}\right) e^{-\theta x}\right)\right]\right\}^{2}}
$$

for $x>0, \theta>0, \lambda>0$, and $0<\pi<1$. We shall refer to the distribution given by (1.7) and (1.8) as the geometric Lindley Poisson (GLP). The parameters, $\lambda$ and $\pi$, control the shape. The parameter, $\theta$, controls the scale. The particular case of (1.6) for $\lambda \rightarrow 0$ is the Lindley geometric (LG) distribution due to Zakerzadeh and Mahmoudi [14]. The particular case for $\pi \rightarrow 0$ is the Lindley Poisson (LP) distribution due to Gui [4].

\subsection{Max Mini Distribution}

Suppose the machine is made of $M$ series units, so that the machine will fail if one of the units fail. Assume that $\mathrm{M}$ is a truncated Poisson random variable independent of $\mathrm{N}$. In addition, the failure times of the units for the $i$ th system, say $Z_{i, 1}, Z_{i, 2}, \ldots, Z_{i, M}$, are independent and identical Lindley random variables with the scale Parameter $\theta$. Let $Y_{i}$ denote the failure time of the $i^{\text {th }}$ system. Let $X$ denote the time to failure of the last of the $N$ functioning systems. We can write $X=\max \left(Y_{1}, Y_{2}, \ldots, Y_{n}\right)$. Then, the 
cumulative distribution function of $X$, say $G(x)$, can be derived as follows. The conditional cumulative distribution function of $X$ given $\mathrm{N}$ is,

$$
G(x)=\frac{(1-\pi) \operatorname{Pr}(Y \leqslant x)}{1-\pi \operatorname{Pr}(Y \leqslant x)}
$$

and

so

$$
\operatorname{Pr}(Y \leqslant x)=\operatorname{Pr}\left[\left(Z_{i, 1}, Z_{i, 2}, \ldots, Z_{i, M}\right) \leqslant x\right]=\frac{\exp \left(\lambda-\lambda\left(\frac{\theta+1+\theta x}{1+\theta}\right) e^{-\theta x}\right)}{e^{\lambda}-1}
$$

$$
G(x)=\frac{(1-\pi)\left[1-\exp \left(-\lambda+\lambda\left(\frac{\theta+1+\theta x}{1+\theta}\right) e^{-\theta x}\right)\right]}{1-e^{-\lambda}-\pi\left[1-\exp \left(-\lambda+\lambda\left(\frac{\theta+1+\theta x}{1+\theta}\right) e^{-\theta x}\right)\right]},
$$

for $x>0, \theta>0, \lambda>0$, and $0<\pi<1$. The corresponding probability density function is,

$$
g(x)=\frac{\lambda \theta^{2}(1-\pi)\left(1-e^{-\lambda}\right)(1+x) \exp \left(-\theta x-\lambda+\lambda\left(\frac{\theta+1+\theta x}{1+\theta}\right) e^{-\theta x}\right)}{(1+\theta)\left\{1-e^{-\lambda}-\pi\left[1-\exp \left(-\lambda+\lambda\left(\frac{\theta+1+\theta x}{1+\theta}\right) e^{-\theta x}\right)\right]\right\}^{2}} .
$$

We shall refer to the distribution given by (1.8) and (1.9) as the geometric Lindley Poisson (GLP1). The parameters $\lambda$ and $\pi$ control the shape. The parameter $\theta$ controls the scale.

Sankaran [10] introduced the discrete Poisson Lindley distribution by compounding Poission and Lindley distributions. Ghitany et al. [3] investigated the properties of the zero-truncated Poisson-Lindley distribution. Bakouch et al. [1] extended. Lindley distribution by exponentiation. Zakerzadeh and Dolati [13] introduced and analyzed a three-parameter generalization of the Lindley distribution, which was used by Mahmoudi and Zakerzadeh [5] to derive an extended version of the compound Poisson distribution. Shanker et al. [11] introduced a two-parameter Lindley distribution in which the one-parameter is a particular case, for modeling waiting and survival time data. Ghitany et al. [3] introduced a twoparameter power Lindley distribution (PL) and discussed its properties. Nadarajah et al. [7] proposed a generalized Lindley distribution (GL) and provided a comprehensive account of its mathematical properties.

The failure rate function associated with (1.6) is given by

$$
h(x)=\frac{\lambda \theta^{2}\left(1-e^{-\lambda}\right)(1+x) \exp \left(-\theta x-\lambda\left(\frac{\theta+1+\theta x}{1+\theta}\right) e^{-\theta x}\right)}{(1+\theta)\left\{1-e^{-\lambda}-\pi\left[1-\exp \left(-\lambda\left(\frac{\theta+1+\theta x}{1+\theta}\right) e^{-\theta x}\right)\right]\right\}} \frac{1}{\left(1-\exp \left(-\lambda\left(\frac{\theta+1+\theta x}{1+\theta}\right) e^{-\theta x}\right)\right)} .
$$

Also, the reversed failure rate function

$$
\operatorname{rh}(x)=\frac{\lambda \theta^{2}(1-\pi)\left(1-e^{-\lambda}\right)(1+x) \exp \left(-\theta x-\lambda\left(\frac{\theta+1+\theta x}{1+\theta}\right) e^{-\theta x}\right)}{(1+\theta)\left[\exp \left(-\lambda\left(\frac{\theta+1+\theta x}{1+\theta}\right) e^{-\theta x}\right)-e^{-\lambda}\right]\left\{1-e^{-\lambda}-\pi\left[1-\exp \left(-\lambda e^{-(\beta x)^{\alpha}}\right)\right]\right\}} .
$$

Figure 1 ((a) and (b)) provides some plots of the GLP density curves for different values of the parameters $\lambda, \theta$, and $\pi$.

Figure 2 does the same for the associated hazard rate function, showing that it is quite flexible for modelling survival data.

Note that $h(x) \rightarrow \theta$ as $x \rightarrow \infty$, and $h(x) \rightarrow \lambda \theta^{2} e^{-\lambda} /\left(1-e^{-\lambda}\right)(1+\theta)(1+\pi)$ as $x \rightarrow 0$. So, both the initial and ultimate hazard rates are constant.

The rest of the paper is organized as follows. In Section 2, we derive an expansion to the pdf and the cdf functions. Section 3, gives the quantile function for the new model. In Section 4, some properties of the new distribution are given. Bonferroni and Lorenz Curves and mean deviations are discussed in Section 5. In Section 6, we introduce the method of likelihood estimation as point estimation of the unknown parameters. Section 7 contains measures of uncertainty. In Section 8, we fit the distribution to two real data sets to examine it and to suitability it with nested and non-nested models. Section 9, describes a generalization of the proposed model. 


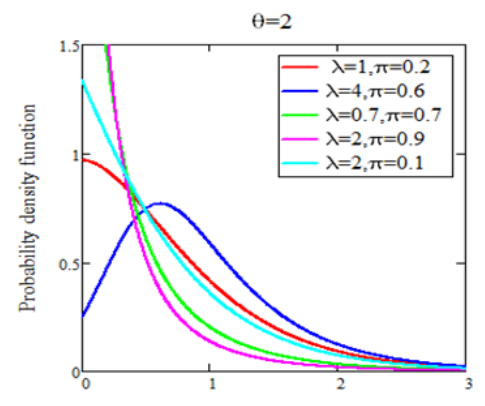

(a)

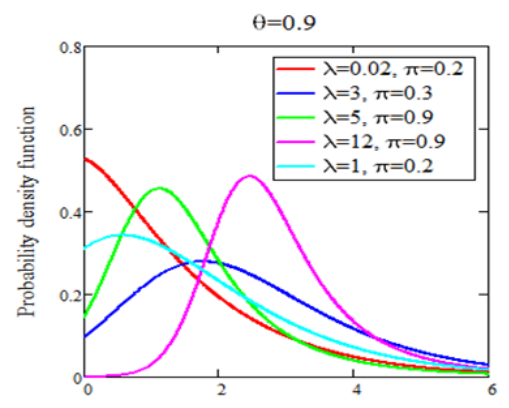

(b)

Figure 1: Plots of the GLP density function for some parameter values.

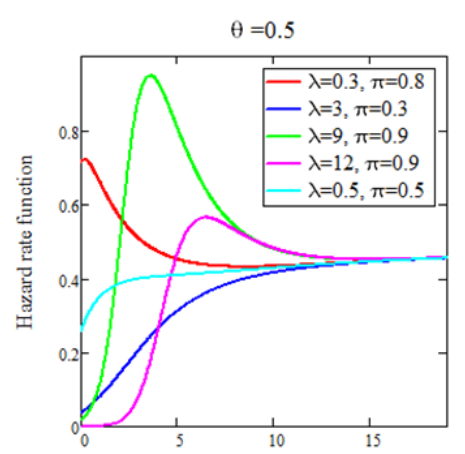

(a)

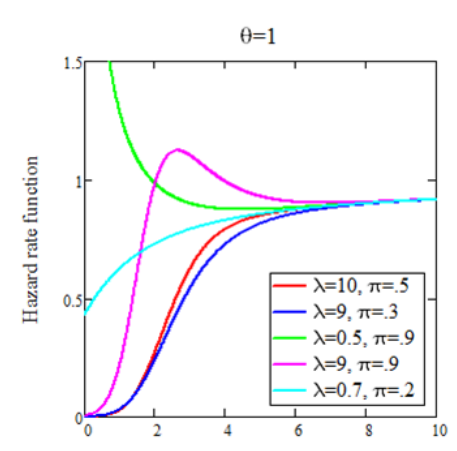

(b)

Figure 2: Plots of the GLP hazard rate function for some parameter values.

\section{Expansion for the pdf and the cdf functions}

In this section we give another expression for the pdf and the cdf functions using the Maclaurin and Binomial expansions for simplifying the pdf and the cdf forms.

\subsection{Expansion for the pdf function}

We can rewrite (1.6) as,

$$
g(x)=\frac{\lambda \theta^{2}(1-\pi)\left(1-e^{-\lambda}\right)(1+x) \exp \left(-\theta x-\lambda\left(\frac{\theta+1+\theta x}{1+\theta}\right) e^{-\theta x}\right)}{(1+\theta)\left(1-e^{-\lambda}-\pi\right)^{2}\left[1+\frac{\pi \exp \left(-\lambda\left(\frac{\theta+1+\theta x}{1+\theta}\right) e^{-\theta x}\right)}{1-e^{-\lambda}-\pi}\right]^{2}}
$$

Using the expansions

$$
(1-z)^{-b}=\sum_{i=0}^{8}\left(\begin{array}{c}
-b \\
i
\end{array}\right)(-z)^{i},|z|<1
$$

and

$$
\mathrm{e}^{-\mathrm{x}}=\sum_{i=0}^{\infty} \frac{(-x)^{i}}{i !}
$$

Using (2.1), we can write (2.2) as

$$
\begin{aligned}
g(x)= & \frac{\lambda \theta^{2}(1-\pi)\left(1-e^{-\lambda}\right)(1+x) \exp (-\theta x)}{(1+\theta)\left(1-e^{-\lambda}-\pi\right)^{2}} \\
& \times \sum_{k=0}^{\infty}\left(\begin{array}{c}
-2 \\
k
\end{array}\right)\left[\frac{\pi}{1-e^{-\lambda}-\pi}\right]^{k} \exp \left(-\lambda(k+1)\left(\frac{\theta+1+\theta x}{1+\theta}\right) e^{-\theta x}\right) .
\end{aligned}
$$


Applying (2.3) to (2.4) for the term $\exp \left(-\lambda(k+1)\left(\frac{\theta+1+\theta x}{1+\theta}\right) e^{-\theta x}\right),(2.4)$ can be written as:

$$
\begin{aligned}
g(x)= & \frac{\lambda \theta^{2}(1-\pi)\left(1-e^{-\lambda}\right)(1+x) \exp (-\theta x)}{(1+\theta)\left(1-e^{-\lambda}-\pi\right)^{2}} \\
& \times \sum_{k, j=0}^{\infty}\left(\begin{array}{c}
-2 \\
k
\end{array}\right) \frac{(-1)^{j}}{j !}\left[\frac{\pi}{1-e^{-\lambda}-\pi}\right]^{k} \lambda^{j}(k+1)^{j}\left(1+\frac{\theta x}{1+\theta}\right)^{j} e^{-\theta j x} .
\end{aligned}
$$

Applying (2.2) to (2.5) for the term $\left(1+\frac{\theta x}{1+\theta}\right)^{j},(2.5)$ can be written as:

$$
g(x)=\frac{\lambda \theta^{2}(1-\pi)\left(1-e^{-\lambda}\right)}{(1+\theta)\left(1-e^{-\lambda}-\pi\right)^{2}} \sum_{k, j=0}^{\infty} \sum_{i=0}^{j}\left(\begin{array}{c}
j \\
i
\end{array}\right)\left(\begin{array}{c}
-2 \\
k
\end{array}\right) \frac{(-1)^{j} \theta^{i}}{j !(1+\theta)^{i}}\left[\frac{\pi}{1-e^{-\lambda}-\pi}\right]^{k} \lambda^{j}(k+1)^{j}(1+x) x^{i} e^{-(j+1) \theta x} .
$$

The pdf of GLP distribution can then be represented as:

$$
g(x)=\sum_{k, j=0}^{\infty} \sum_{i=0}^{j} A_{k: i}(1+x) x^{i} e^{-(j+1) \theta x},
$$

where $A_{k: i}$ is a constant term given by

$$
A_{k: i}=\left(\begin{array}{c}
-2 \\
k
\end{array}\right)\left(\begin{array}{c}
j \\
i
\end{array}\right) \frac{\theta^{2+i}(1-\pi)\left(1-e^{-\lambda}\right)(-1)^{j} \pi^{k} \lambda^{j+1}(k+1)^{j}}{(1+\theta)^{i+1}\left(1-e^{-\lambda}-\pi\right)^{k+2} j !} .
$$

2.2. Expansion for the cdf function

We can rewrite (1.5) as,

$$
G(x)=\frac{\exp \left(-\lambda\left(\frac{\theta+1+\theta x}{1+\theta}\right) e^{-\theta x}\right)-e^{-\lambda}}{\left(1-e^{-\lambda}-\pi\right)\left[1+\frac{\pi \exp \left(-\lambda\left(\frac{\theta+1+\theta x}{1+\theta}\right) e^{-\theta x}\right)}{1-e^{-\lambda}-\pi}\right]} .
$$

And applying the expansion in (2.2), the cdf function of the GLP distribution can be written as:

$$
G(x)=\sum_{k=0}^{\infty}\left(\begin{array}{c}
-1 \\
k
\end{array}\right) \frac{\pi^{k}\left[\exp \left(-(k+1) \lambda\left(\frac{\theta+1+\theta x}{1+\theta}\right) e^{-\theta x}\right)-\exp \left(-\lambda-k \lambda\left(\frac{\theta+1+\theta x}{1+\theta}\right) e^{-\theta x}\right)\right]}{\left(1-e^{-\lambda}-\pi\right)^{k+1}} .
$$

\section{Quantile function}

The quantile function is obtained by inverting the cumulative distribution (1.5), where the $p^{\text {th }}$ quantile $x_{p}$ of the GLP model is the real solution of the following equation:

$$
\ln \left(\theta+1+\theta x_{\mathfrak{p}}\right)-\theta x_{\mathfrak{p}}-\ln \left[\frac{-(1+\theta)}{\lambda} \ln \left(\frac{p(1-\pi)+(1-p) e^{-\lambda}}{1-\pi p}\right)\right]=0 .
$$

An expansion for the median $M$ follows by taking $p=0.5$.

\section{Statistical properties}

In this section, moments, conditional moments, moment generating function of the GLP distribution are presented. 


\subsection{Moments}

The $r^{\text {th }}$ non-central moments or (moments about the origin) of the GLP under using equation (2.6) is given by:

$$
\mu_{r}^{\prime}=E\left(X^{r}\right)=\int_{0}^{\infty} X^{r} g(x) d x, \quad \mu_{r}^{\prime}=\int_{0}^{\infty} X^{r}\left[\sum_{k, j=0}^{\infty} \sum_{i=0}^{j} A_{k: i}(1+x) x^{i} e^{-(j+1) \theta x}\right] d x,
$$

then,

$$
\mu_{r}^{\prime}=\sum_{k, j=0}^{\infty} \sum_{i=0}^{j} A_{k: i} \frac{1}{(\theta(j+1))^{r+i+2}}[\theta(j+1) \Gamma(r+i+1)+\Gamma(r+i+2)]
$$

Thus

$$
\mu_{1}^{\prime}=E(x)=\sum_{k, j=0}^{\infty} \sum_{i=0}^{j} A_{k: i} \frac{1}{(\theta(j+1))^{i+3}}[\theta(j+1) \Gamma(i+2)+\Gamma(i+3)] .
$$

\subsection{Conditional moments}

For lifetime models, it is useful to know the conditional moments defined as $E\left(\chi^{r} \mid x>t\right)$,

$$
E\left(x^{r} \mid x>t\right)=\frac{1}{[1-G(t)]} \int_{t}^{\infty} x^{r} g(x) d x,
$$

using equation (2.6) the conditional moments is,

$$
E\left(x^{r} \mid x>t\right)=\frac{1}{[1-G(t)]} \sum_{k, j=0}^{\infty} \sum_{i=0}^{j} A_{k: i} \frac{1}{(\theta(j+1))^{r+i+2}}\left[\theta(j+1) \Gamma_{t}(r+i+1)+\Gamma_{t}(r+i+2)\right],
$$

where $\Gamma_{t}(a)=\int_{t}^{\infty} x^{a-1} e^{-x} d x$ is the upper incomplete gamma function.

\subsection{The moment generating function}

The moment generating function, $M_{x}(t)$, can be easily obtained as:

$$
M_{x}(t)=\int_{0}^{\infty} e^{t x} g(x) d x, \quad M_{x}(t)=\int_{0}^{\infty} e^{t x} \sum_{k, j=0}^{\infty} \sum_{i=0}^{j} A_{k: i}(1+x) x^{i} e^{-(j+1) \theta x} d x,
$$

then, the moment generating function of the GLP distribution is given by,

$$
M_{x}(t)=\sum_{k, j=0}^{\infty} \sum_{i=0}^{j} \frac{A_{k: i}}{[\theta(j+1)-t]^{i+2}}\{(\theta(j+1)-t) \Gamma(i+1)+\Gamma(i+2)\}
$$

\section{Lorenz curves, Bonferroni, and mean deviations}

In this section, we present Lorenz curves, Bonferroni, and the mean deviation about the mean, and the mean deviation about the median. Bonferroni and Lorenz curves are income inequality measures that are also useful and applicable in other areas including reliability, demography, medicine, and insurance.

\subsection{Lorenz curves and Bonferroni}

The Lorenz curves L (G) and Bonferroni B (G) are defined by

$$
L(G)=\frac{1}{\mu} \int_{0}^{x} \operatorname{tg}(t) d t, \quad L(G)=\sum_{k, j=0}^{\infty} \sum_{i=0}^{j} A_{k: i} \frac{1}{\mu(\theta(j+1))^{i+3}}[\theta(j+1) \Gamma[(i+2), x]+\Gamma[(i+3), x]],
$$


where $\Gamma(a, x)=\int_{0}^{x} t^{a-1} e^{-t} d t$, is the lower incomplete gamma function. And

$$
\begin{aligned}
& B(G)=\frac{1}{\mu G(x)} \int_{0}^{x} \operatorname{tg}(t) d t \\
& B(G)=\sum_{k, j=0}^{\infty} \sum_{i=0}^{j} A_{k: i} \frac{1}{\mu G(x)(\theta(j+1))^{i+3}}[\theta(j+1) \Gamma[(i+2), x]+\Gamma[(i+3), x]] .
\end{aligned}
$$

\subsection{The mean deviation}

In statistics, mean deviation about the mean and mean deviation about the median measure the amount of scatter in a population. For random variable $X$ with pdf $g(t)$, distribution function $G(x)$, mean $\mu$, and $M=$ Median $(X)$, the mean deviation about the mean and mean deviation about the median, are defined by,

$$
\delta_{1}(x)=\int_{0}^{\infty}|x-\mu| g(x) d x=2 \mu G(\mu)-2 \int_{0}^{\mu} x g(x) d x, \quad \text { and } \quad \delta_{2}(x)=\int_{0}^{\infty}|x-M| g(x) d x=\mu-2 \int_{0}^{M} x g(x) d x,
$$

respectively, if $X$ is GLP random variable then

$$
\delta_{1}(x)=2 \mu G(x)-2 \sum_{k, j=0}^{\infty} \sum_{i=0}^{j} A_{k: i} \frac{1}{((j+1) \theta)^{i+3}}[(j+1) \theta \Gamma[(i+2), \mu]+\Gamma[(i+3), \mu]],
$$

and

$$
\delta_{2}(x)=\mu-2 \sum_{k, j=0}^{\infty} \sum_{i=0}^{j} A_{k: i} \frac{1}{((j+1) \theta)^{i+3}}[(j+1) \theta \Gamma[(i+2), M]+\Gamma[(i+3), M]] .
$$

\section{Measures of uncertainty}

In this section, we present Shannon entropy [12], as well as Re'nyi entropy (1961) for the GLP distribution. The concept of entropy plays a vital role in information theory. The entropy of a random variable is defined in terms of its probability distribution and is a good measure of randomness or uncertainty.

\subsection{Shannon entropy}

Shannon entropy [12], is defined by $\mathrm{H}[\mathrm{g}(\mathrm{x})]=\mathrm{E}[-\ln [\mathrm{g}(\mathrm{x})]]$. Thus, using (2.1) we have

$$
\begin{aligned}
H[g(x)]= & -\ln \left[\frac{\lambda \theta^{2}(1-\pi)\left(1-e^{-\lambda}\right)}{(1+\theta)\left(1-e^{-\lambda}-\pi\right)^{2}}\right]-E \ln (1+x)+\theta E(x)+\lambda E\left[\left(\frac{\theta+1+\theta x}{1+\theta}\right) \exp (-\theta x)\right] \\
& +2 E \ln \left[1+\frac{\pi \exp \left(-\lambda\left(\frac{\theta+1+\theta x}{1+\theta}\right) e^{-\theta x}\right)}{\left(1-e^{-\lambda}-\pi\right)}\right] .
\end{aligned}
$$

Note that,

$$
E \ln \left(1+x^{a}\right)=-\sum_{q=1}^{\infty} \frac{(-1)^{q}}{q} E\left(x^{q a}\right)
$$

Using (6.1),

$$
\operatorname{Eln}\left[1+\frac{\pi \exp \left(-\lambda\left(\frac{\theta+1+\theta x}{1+\theta}\right) e^{-\theta x}\right)}{\left(1-e^{-\lambda}-\pi\right)}\right]=-\sum_{q=1}^{\infty} \frac{(-\pi)^{q} E\left(\exp \left(-\lambda q\left(\frac{\theta+1+\theta x}{1+\theta}\right) e^{-\theta x}\right)\right)}{q\left(1-e^{-\lambda}-\pi\right)^{q}}
$$




$$
=-\sum_{q=1}^{\infty} \sum_{s=0}^{\infty} \sum_{r=1}^{s}\left(\begin{array}{c}
s \\
r
\end{array}\right) \frac{(-1)^{q+s} \pi^{q} \lambda^{s} \theta^{r} E\left(\chi^{r}\left(e^{-\theta s x}\right)\right)}{s ! q^{1-s}\left(1-e^{-\lambda}-\pi\right)^{q}(1+\theta)^{r}}
$$

then,

$$
\begin{aligned}
H[g(x)]= & -\ln \left[\frac{\lambda \theta^{2}(1-\pi)\left(1-e^{-\lambda}\right)}{(1+\theta)\left(1-e^{-\lambda}-\pi\right)^{2}}\right]+\sum_{l=1}^{\infty} \frac{(-1)^{r}}{r} E\left(x^{r}\right)+\theta E(x)+\lambda E\left(e^{-\theta x}\right)+\frac{\lambda \theta}{1+\theta} E\left(x e^{-\theta x}\right) \\
& -2 \sum_{q=1}^{\infty} \sum_{s=0}^{\infty} \sum_{r=1}^{s}\left(\begin{array}{c}
s \\
r
\end{array}\right) \frac{(-1)^{q+s} \pi^{q} \lambda^{s} \theta^{r} E\left(x^{r}\left(e^{-\theta s x}\right)\right)}{s ! q^{1-s}\left(1-e^{-\lambda}-\pi\right)^{q}(1+\theta)^{r}}
\end{aligned}
$$

Now, we obtain Shannon entropy for the GLP distribution as follows:

$$
\begin{aligned}
& H[g(x)]=-\ln \left[\frac{\lambda \theta^{2}(1-\pi)\left(1-e^{-\lambda}\right)}{(1+\theta)\left(1-e^{-\lambda}-\pi\right)^{2}}\right]+\sum_{l=1}^{\infty} \frac{(-1)^{r}}{r} \sum_{k, j=0}^{\infty} \sum_{i=0}^{j} \frac{A_{k: i}}{[(j+1) \theta]^{r+i+2}}\{((j+1) \theta) \Gamma(r+i+1)+\Gamma(r+i+2)\} \\
& +\theta \sum_{k, j=0}^{\infty} \sum_{i=0}^{j} \frac{A_{k: i}}{[(j+1) \theta]^{i+3}}\{((j+1) \theta) \Gamma(i+2)+\Gamma(i+3)\} \\
& +\lambda \sum_{k, j=0}^{\infty} \sum_{i=0}^{j} \frac{A_{k: i}}{[(j+2) \theta]^{i+2}}\{((j+2) \theta) \Gamma(i+1)+\Gamma(i+2)\} \\
& +\frac{\lambda \theta}{1+\theta} \sum_{k, j=0}^{\infty} \sum_{i=0}^{j} \frac{A_{k: i}}{[(j+2) \theta]^{i+3}}\{((j+2) \theta) \Gamma(i+2)+\Gamma(i+3)\} \\
& -2 \sum_{\mathrm{q}=1}^{\infty} \sum_{s=0}^{\infty} \sum_{r=1}^{s}\left(\begin{array}{l}
s \\
r
\end{array}\right) \frac{(-1)^{\mathrm{q}+s} \pi^{\mathrm{q}} \lambda^{s} \theta^{\mathrm{r}}}{s ! q^{1-s}\left(1-e^{-\lambda}-\pi\right)^{\mathrm{q}}(1+\theta)^{\mathrm{r}}} \\
& \times\left[\sum_{k, j=0}^{\infty} \sum_{i=0}^{j} \frac{A_{k: i}}{[(j+s+1) \theta]^{r+i+2}}\{((j+s+1) \theta) \Gamma(r+i+1)+\Gamma(r+i+2)\}\right] .
\end{aligned}
$$

\subsection{Re'nyi entropy}

Re'nyi entropy [9] is an extension of Shannon entropy. Re'nyi entropy is defined to be

$$
I_{R}(r)=\frac{1}{1-r} \ln \left[\int_{0}^{\infty} g^{r}(x) d x\right], \quad r>0, \quad r \neq 1,
$$

where

$$
\begin{aligned}
g^{r}(x)= & {\left[\frac{\lambda \theta^{2}(1-\pi)\left(1-e^{-\lambda}\right)}{(1+\theta)\left(1-e^{-\lambda}-\pi\right)^{2}}\right]^{r}(1+x)^{r} \exp \left(-\theta r x-\lambda r\left(\frac{\theta+1+\theta x}{1+\theta}\right) e^{-\theta x}\right) } \\
& \times\left[1+\frac{\exp \left(-\lambda\left(\frac{\theta+1+\theta x}{1+\theta}\right) e^{-\theta x}\right)}{\left(1-e^{-\lambda}-\pi\right)}\right]^{-2 r},
\end{aligned}
$$

we can write (6.3) as:

$$
\begin{aligned}
g^{r}(x)= & {\left[\frac{\lambda \theta^{2}(1-\pi)\left(1-e^{-\lambda}\right)}{(1+\theta)\left(1-e^{-\lambda}-\pi\right)^{2}}\right]^{r} \sum_{k, j=0}^{\infty} \sum_{i, l=0}^{j, r}\left(\begin{array}{c}
-2 r \\
k
\end{array}\right)\left(\begin{array}{l}
j \\
i
\end{array}\right)\left(\begin{array}{l}
r \\
l
\end{array}\right) \frac{\pi^{k}}{\left(1-e^{-\lambda}-\pi\right)^{k}} } \\
& \times \frac{(-1)^{j} \lambda^{j}(r+k)^{j} \theta^{i}}{(1+\theta)^{i}} x^{i+l} e^{-(j+r) \theta x} .
\end{aligned}
$$


The $g^{r}(x)$ of GLP1 distribution can then be represented as:

$$
g^{r}(x)=\sum_{k, j=0}^{\infty} \sum_{i, l=0}^{j, r} B_{k: l} x^{i+l} e^{-(j+r) \theta x}
$$

where $B_{k: l}$ is a constant term given by

$$
B_{k: l}=\left[\lambda(1-\pi)\left(1-e^{-\lambda}\right)\right]^{r}\left(\begin{array}{c}
-2 r \\
k
\end{array}\right)\left(\begin{array}{l}
j \\
i
\end{array}\right)\left(\begin{array}{l}
r \\
l
\end{array}\right) \frac{\pi^{k}(-1)^{j} \lambda^{j}(r+k)^{j} \theta^{2 r+i}}{\left(1-e^{-\lambda}-\pi\right)^{2 r+k}(1+\theta)^{r+i}} .
$$

Then

$$
\int_{0}^{\infty} g^{r}(x) d x=\sum_{k, j=0}^{\infty} \sum_{i, l=0}^{j, r} B_{k: l} \int_{0}^{\infty} x^{i+l} e^{-(j+r) \theta x} d x,=\sum_{k, j=0}^{\infty} \sum_{i, l=0}^{j, r} B_{k: l} \frac{\Gamma(i+l+1)}{[(j+r) \theta]^{i+l+1}},
$$

by substituting (6.4) in (6.2),

$$
I_{R}(r)=\frac{1}{1-r} \ln \left[\sum_{k, j=0}^{\infty} \sum_{i, l=0}^{j, r} B_{k: l} \frac{\Gamma(i+l+1)}{[(j+r) \theta]^{i+l+1}}\right], \quad r>0, \quad r \neq 1 .
$$

\section{3. s-entropy}

The s-entropy for the GLP distribution is defined by

$$
\mathrm{I}_{\mathrm{s}}(\mathrm{r})=\frac{1}{\mathrm{~s}-1}\left[1-\int_{0}^{\infty} \mathrm{g}^{\mathrm{s}}(\mathrm{x}) \mathrm{d} x\right], \quad s>0, \quad s \neq 1 .
$$

Now, using the same procedure that was used to derive equation (6.5), we have

$$
I_{s}(r)=\frac{1}{s-1}\left[1-\sum_{k, j=0}^{\infty} \sum_{i, l=0}^{j, s} D_{k: l} \frac{\Gamma(i+l+1)}{[(j+s) \theta]^{i+l+1}}\right], \quad s>0, \quad s \neq 1,
$$

where $D_{k: l}$ is a constant term given by

$$
D_{k: l}=\left[\lambda(1-\pi)\left(1-e^{-\lambda}\right)\right]^{s}\left(\begin{array}{c}
-2 s \\
k
\end{array}\right)\left(\begin{array}{l}
j \\
i
\end{array}\right)\left(\begin{array}{l}
s \\
l
\end{array}\right) \frac{\pi^{k}(-1)^{j} \lambda^{j}(s+k)^{j} \theta^{2 s+i}}{\left(1-e^{-\lambda}-\pi\right)^{2 s+k}(1+\theta)^{s+i}} .
$$

\section{Parameter estimation}

In this section, the maximum likelihood estimation is used to estimate the unknown parameters. Let $X_{1}, X_{2}, \ldots, X_{n}$ be a sample of size $n$ from a GLP distribution. Then the likelihood function $(\ell)$ is given by

$$
\ell=\frac{\lambda^{n} \theta^{2 n}(1-\pi)^{n}\left(1-e^{-\lambda}\right)^{n} \prod_{i=1}^{n}\left(1+x_{i}\right) \exp \left(-\theta \sum_{i=1}^{n} x_{i}-\lambda \sum_{i=1}^{n}\left(\frac{\theta+1+\theta x_{i}}{1+\theta}\right) e^{-\theta x_{i}}\right)}{(1+\theta)^{n} \prod_{i=1}^{n}\left\{1-e^{-\lambda}-\pi\left[1-\exp \left(-\lambda\left(\frac{\theta+1+\theta x_{i}}{1+\theta}\right) e^{-\theta x_{i}}\right)\right]\right\}^{2}} .
$$

Hence, the log-likelihood function, $\mathcal{L}$, becomes:

$$
\begin{aligned}
\mathcal{L}= & n \ln \lambda+2 n \ln \theta+n \ln (1-\pi)+n \ln \left(1-e^{-\lambda}\right) \\
& -n \ln (1+\theta)+\sum_{i=1}^{n}\left(1+x_{i}\right)-\theta \sum_{i=1}^{n} x_{i}-\lambda \sum_{i=1}^{n}\left(\frac{\theta+1+\theta x_{i}}{1+\theta}\right) e^{-\theta x_{i}} \\
& -2 \sum_{i=1}^{n} \ln \left[1-e^{-\lambda}-\pi\left[1-\exp \left(-\lambda\left(\frac{\theta+1+\theta x_{i}}{1+\theta}\right) e^{-\theta x_{i}}\right)\right]\right] .
\end{aligned}
$$


Therefore, the MLEs of $\lambda, \theta$, and $\pi$ must satisfy the following equations:

$$
\begin{aligned}
& \frac{\partial \mathcal{L}}{\partial \lambda}= \frac{n}{\lambda}+\frac{n}{e^{-\lambda}-1}-\sum_{i=1}^{n}\left(\frac{\theta+1+\theta x_{i}}{1+\theta}\right) e^{-\theta x_{i}} \\
&-2 \sum_{i=1}^{n} \frac{e^{-\lambda}-\pi\left(\frac{\theta+1+\theta x_{i}}{1+\theta}\right) \exp \left(-\theta x_{i}-\lambda\left(\frac{\theta+1+\theta x_{i}}{1+\theta}\right) e^{-\theta x_{i}}\right)}{\left[1-e^{-\lambda}-\pi\left[1-\exp \left(-\lambda\left(\frac{\theta+1+\theta x_{i}}{1+\theta}\right) e^{-\theta x_{i}}\right)\right]\right]}, \\
& \frac{\partial \mathcal{L}}{\partial \theta}=\frac{2 n}{\theta}-\frac{n}{1+\theta}-\sum_{i=1}^{n} x_{i}+\frac{\lambda \theta}{(1+\theta)^{2}} \sum_{i=1}^{n}\left[(2+\theta) x_{i}+(1+\theta) x_{i}^{2}\right] e^{-\theta x_{i}} \\
&-\sum_{i=1}^{n} \frac{2 \lambda \theta \pi \exp \left(-\theta x_{i}-\lambda\left(\frac{\theta+1+\theta x_{i}}{1+\theta}\right) e^{-\theta x_{i}}\right)\left[(2+\theta) x_{i}+(1+\theta) x_{i}^{2}\right]}{(1+\theta)^{2}\left[1-e^{-\lambda}-\pi\left[1-\exp \left(-\lambda\left(\frac{\theta+1+\theta x_{i}}{1+\theta}\right) e^{-\theta x_{i}}\right)\right]\right]}
\end{aligned}
$$

and

$$
\frac{\partial \mathcal{L}}{\partial \pi}=\frac{-n}{(1-\pi)}+2 \sum_{i=1}^{n} \frac{1-\exp \left(-\lambda\left(\frac{\theta+1+\theta x_{i}}{1+\theta}\right) e^{-\theta x_{i}}\right)}{1-e^{-\lambda}-\pi\left[1-\exp \left(-\lambda\left(\frac{\theta+1+\theta x_{i}}{1+\theta}\right) e^{-\theta x_{i}}\right)\right]}
$$

The maximum likelihood estimator $\underline{\hat{\vartheta}}=(\hat{\lambda}, \widehat{\theta}, \widehat{\pi})$ of $\underline{\vartheta}=(\lambda, \theta, \pi)$ is obtained by solving the nonlinear system of equations (7.3)-(7.5). It is usually more convenient to use nonlinear optimization algorithms such as quasi-Newton algorithm to numerically maximize the log-likelihood function.

\section{Application}

In this section, we use two real data sets to show that the geometric Lindley Poisson (GLP) distribution can be a better model than nested and non-nested models.

\subsection{Data Set 1}

The data set represents an uncensored data set corresponding to remission times (in months) of a random sample of 128 bladder cancer patients reported in Merovci [6]. Some summary statistics for the data are as follows:

$$
\begin{array}{cccccc}
\text { Min } & \text { 1st Qu } & \text { Median } & \text { Mean } & \text { 3rd Qu } & \text { Max. } \\
0.080 & 3.348 & 6.395 & 9.366 & 11.840 & 79.05
\end{array}
$$

In order to compare the two distribution models, we consider criteria like KS (Kolmogorov Smirnov), $-2 \mathcal{L}$, AIC (Akaike information criterion), BIC and AICC (corrected Akaike information criterion) for the data set. The better distribution corresponds to smaller KS,-2L, AIC, and AICC values:

$$
\mathrm{AIC}=-2 \mathcal{L}+2 \mathrm{k}, \quad \mathrm{AIC}_{\mathrm{C}}=-2 \mathcal{L}+\left(\frac{2 \mathrm{kn}}{\mathrm{n}-\mathrm{k}-1}\right)
$$

and BIC $=-2 \mathcal{L}+k \log (n)$, where $\mathcal{L}$ denotes the log-likelihood function evaluated at the maximum likelihood estimates, $\mathrm{k}$ is the number of parameters, and $\mathrm{n}$ is the sample size.

Also, for calculating the values of KS we use the sample estimates of $\lambda, \theta$, and $\pi$. Table 1 shows the 
parameter estimation based on the maximum likelihood, and gives the values of the criteria AIC, AICC and KS test. The values in Table 1 indicate that the GLP distribution is a strong competitor to other distributions used here for fitting data.

Table 1: MLEs the measures AIC, AICC, BIC, and KS test to data for the models.

\begin{tabular}{|c|c|c|c|c|c|c|c|}
\hline Model & Parameter Estimates & Standard Error & $-2 \mathcal{L}$ & AIC & $\mathrm{AIC}_{\mathrm{C}}$ & BIC & KS \\
\hline \multirow{3}{*}{ GLP } & $\lambda=3.05690$ & 1.2104 & \multirow{3}{*}{409.3017} & \multirow{3}{*}{824.603} & \multirow{3}{*}{824.796} & \multirow{3}{*}{833.15} & \multirow{3}{*}{0.02797} \\
\hline & $\theta=0.05405$ & 0.0229 & & & & & \\
\hline & $\pi=0.99023$ & 0.01019 & & & & & \\
\hline \multirow{3}{*}{ GEP } & $\lambda=0.06366$ & 0.02249 & \multirow{3}{*}{410.2873} & \multirow{3}{*}{826.574} & \multirow{3}{*}{826.768} & \multirow{3}{*}{835.13} & \multirow{3}{*}{0.03847} \\
\hline & $\theta=4.94519$ & 1.1226 & & & & & \\
\hline & $\eta=0.9679$ & 0.0380 & & & & & \\
\hline \multirow{2}{*}{ Transmuted Lindley } & $\lambda=0.61687$ & 0.1688 & \multirow{2}{*}{415.155} & \multirow{2}{*}{834.3101} & \multirow{2}{*}{834.4061} & \multirow{2}{*}{840.01} & \multirow{2}{*}{0.226523} \\
\hline & $\theta=0.1557$ & 0.0150 & & & & & \\
\hline \multirow{2}{*}{ Exponentiated Lindley } & $\alpha=0.1648$ & 0.01664 & \multirow{2}{*}{416.285} & \multirow{2}{*}{836.5719} & \multirow{2}{*}{836.6679} & \multirow{2}{*}{842.27} & \multirow{2}{*}{0.092791} \\
\hline & $\theta=0.733$ & 0.0912 & & & & & \\
\hline Lindley & $\theta=0.1960$ & 0.01234 & 419.529 & 841.0598 & 841.0916 & 843.91 & 0.116398 \\
\hline \multirow{2}{*}{ Power Lindley } & $\theta=0.29432$ & 0.03706 & \multirow{2}{*}{413.353} & \multirow{2}{*}{830.707} & \multirow{2}{*}{830.803} & \multirow{2}{*}{836.41} & \multirow{2}{*}{0.06822} \\
\hline & $\beta=0.83020$ & 0.04722 & & & & & \\
\hline \multirow{3}{*}{ EPL } & $\lambda=0.81907$ & 0.31513 & \multirow{3}{*}{410.4335} & & & \multirow{3}{*}{835.42} & \\
\hline & $\theta=0.56634$ & 0.10279 & & & & & \\
\hline & $\alpha=2.76834$ & 1.30424 & & & & & \\
\hline \multirow{2}{*}{ Weighted Lindley } & $\alpha=0.15945$ & 0.0172 & 416442 & 8368845 & 8369805 & 84258 & 0092567 \\
\hline & $\theta=0.6827$ & 0.1115 & 410.442 & 036.0045 & 800 & 842.00 & 0.092068 \\
\hline & $\theta=6.2675$ & 3.16122 & & & & & \\
\hline Modified Weibull & $\delta=6.3551$ & 3.1869 & 413.969 & 833.9393 & 834.1329 & 837.64 & 0.073875 \\
\hline & $\alpha=1.001$ & 0.0017 & & & & & \\
\hline Exponential & $\theta=0.10677$ & 0.00944 & 414.3419 & 830.683 & 830.715 & 833.53 & \\
\hline
\end{tabular}

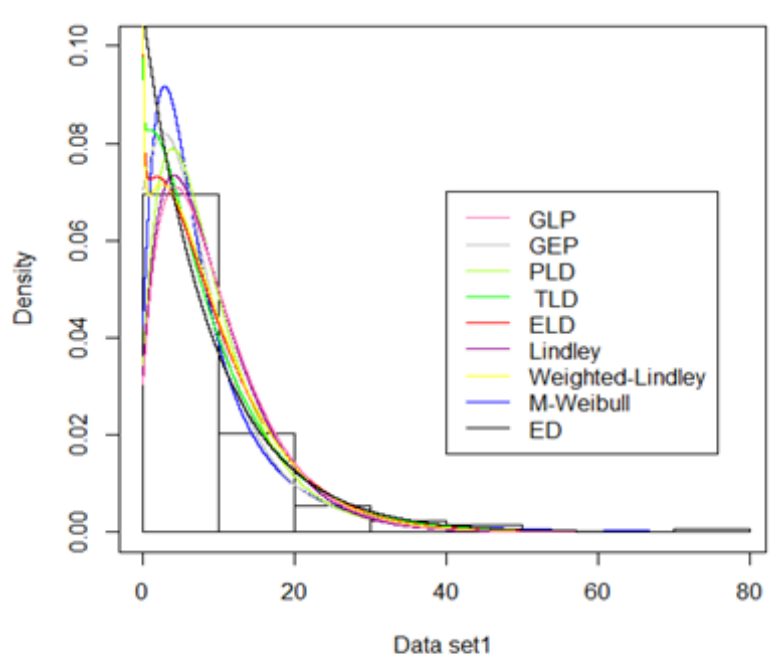

(a)

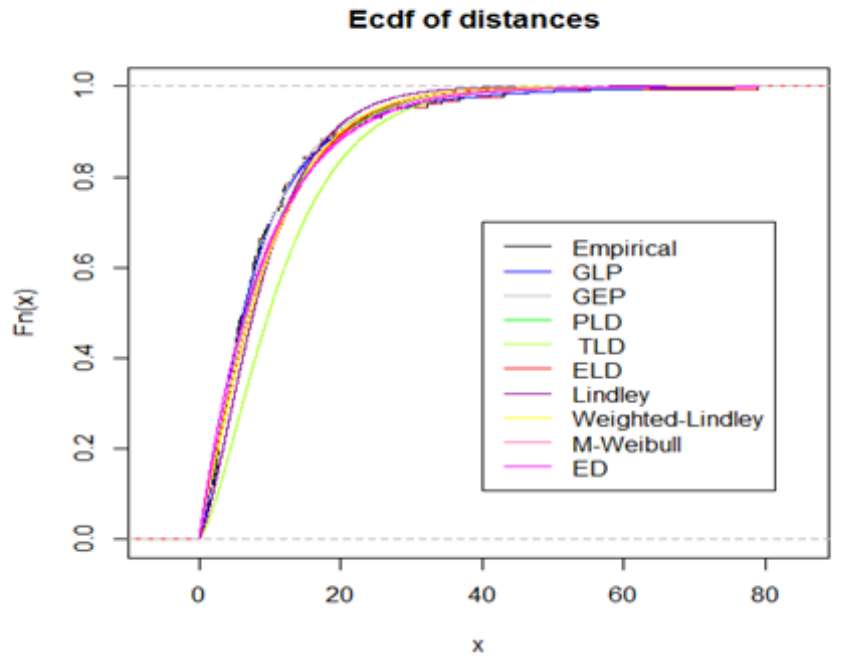

(b)

Figure 3: (a) Estimated densities of the GLP, GEP, PLD, TLD, ELD, LD, WL, MW and Exponential distributions for the data set 1. (b) Estimated cdf function from the fitted the GLP, GEP, PLD, TLD, ELD, LD, WL, MW and Exponential distributions and the empirical cdf for the data set 1. 

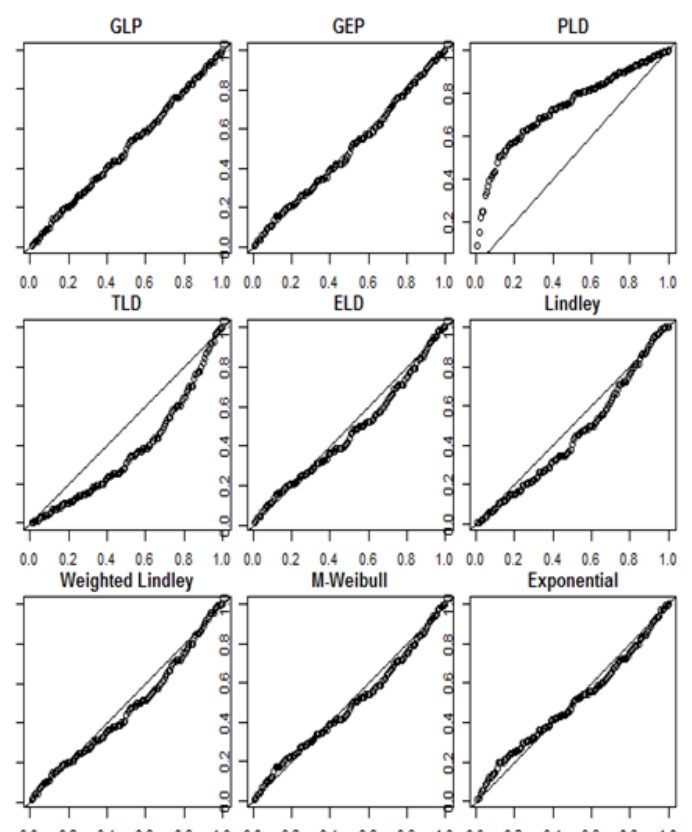

Figure 4: Probability plots for the fits GLP, GEP, PLD, TLD, ELD, LD, WL, MW and Exponential distributions of data set 1.

\subsection{Data Set 2}

The following data represent the survival times (in days) of 72 guinea pigs infected with virulent tubercle bacilli, observed and reported by Bjerkedal [2]. Some summary statistics for the failure time data are as follows:

Min. $1^{\text {st }} \mathrm{Qu} . \quad$ Median $\quad$ Mean $3^{\text {rd }} \mathrm{Qu} . \quad$ Max.

$\begin{array}{llllll}0.10 & 1.080 & 1.495 & 1.768 & 2.240 & 5.550\end{array}$

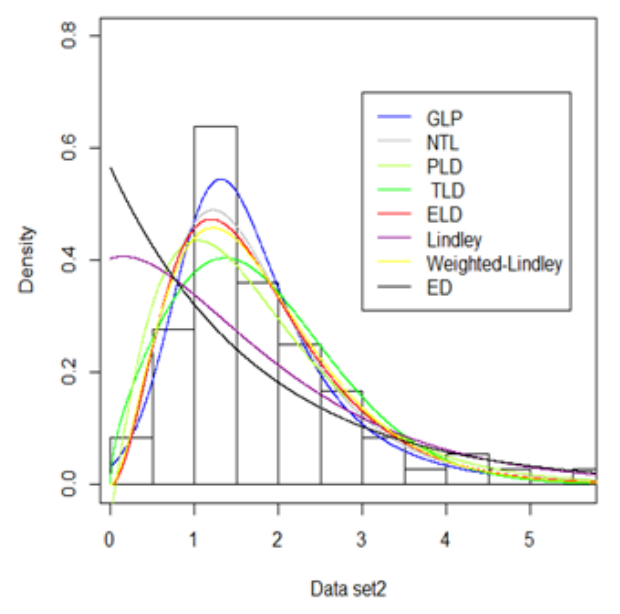

(a)

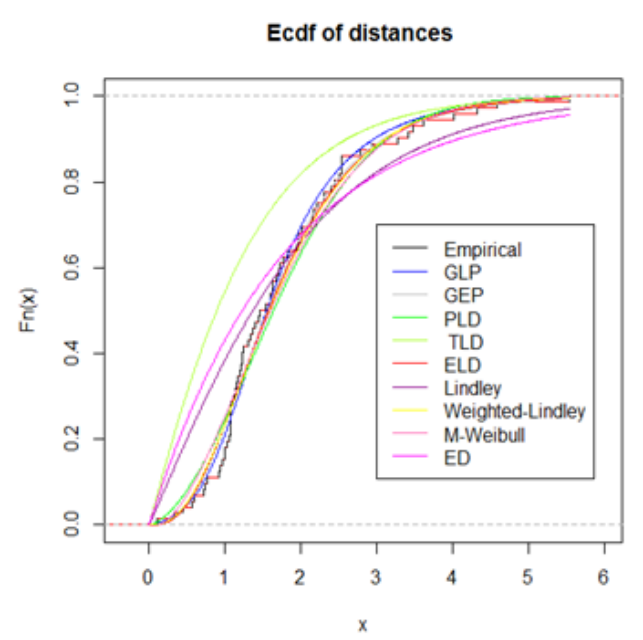

(b)

Figure 5: (a) Estimated densities of the GLP, NTL, PLD, ELD, LD, WL and Exponential distributions for the data set 2. (b) Estimated cdf function from the fitted the GLP, NTL, PLD, TLD, ELD, LD, WL, MW and Exponential distributions and the empirical cdf for the data set 2. 
Table 2: MLEs the measures AIC, AICC, BIC, and KS test to data for the models.

\begin{tabular}{|c|c|c|c|c|c|c|c|}
\hline Model & Parameter Estimates & Standard Error & $-2 \mathcal{L}$ & AIC & $\mathrm{AIC}_{\mathrm{C}}$ & BIC & KS \\
\hline \multirow{3}{*}{ GLP } & $\lambda=6.6408$ & 1.20213 & \multirow{3}{*}{92.572} & \multirow{3}{*}{191.145} & \multirow{3}{*}{191.498} & \multirow{3}{*}{197.97} & \multirow{3}{*}{0.0715} \\
\hline & $\theta=1.2046$ & 0.3100 & & & & & \\
\hline & $\pi=0.8256$ & 0.20720 & & & & & \\
\hline \multirow{4}{*}{ NTL } & $\lambda=0.41674$ & 0.33052 & \multirow{4}{*}{93.650} & \multirow{4}{*}{195.301} & \multirow{4}{*}{195.898} & \multirow{4}{*}{200.13} & \multirow{4}{*}{0.0752} \\
\hline & $\alpha=1.2004$ & 1.3854 & & & & & \\
\hline & $\eta=2.8376$ & 0.2683 & & & & & \\
\hline & $\theta=6.4982$ & & & & & & \\
\hline \multirow{2}{*}{ Transmuted Lindley } & $\lambda=0.0868$ & 0.0219 & \multirow{2}{*}{95.343} & \multirow{2}{*}{194.686} & \multirow{2}{*}{194.860} & \multirow{2}{*}{199.23} & \multirow{2}{*}{0.3875} \\
\hline & $\theta=1.2268$ & 0.08735 & & & & & \\
\hline \multirow{2}{*}{ Exponentiated Lindley } & $\alpha=1.4117$ & 0.14379 & \multirow{2}{*}{93.972} & \multirow{2}{*}{191.945} & \multirow{2}{*}{192.119} & \multirow{2}{*}{196.49} & \multirow{2}{*}{0.0740} \\
\hline & $\theta=2.9647$ & 0.6042 & & & & & \\
\hline Lindley & $\theta=0.86826$ & 0.0766 & 106.928 & 215.856 & 215.914 & 218.13 & 0.2327 \\
\hline \multirow{2}{*}{ Power Lindley } & $\theta=0.59970$ & 0.07594 & \multirow{2}{*}{96.050} & \multirow{2}{*}{196.101} & \multirow{2}{*}{196.275} & \multirow{2}{*}{200.65} & \multirow{2}{*}{0.0904} \\
\hline & $\beta=1.5343$ & 0.12142 & & & & & \\
\hline \multirow{3}{*}{ EPL } & $\lambda=1.3714$ & 0.5029 & \multirow{3}{*}{93.969} & \multirow{3}{*}{193.938} & & & \\
\hline & $\theta=1.0194$ & 0.2368 & & & 194.291 & 200.76 & 0.0735 \\
\hline & $\alpha=2.8367$ & 1.6123 & & & & & \\
\hline Weiohted I indley & $\alpha=1.9048$ & 0.3006 & 04212 & 102424 & 102500 & 10607 & 00753 \\
\hline Weighted Lindley & $\theta=2.7751$ & 0.4818 & 94.212 & 192.424 & 192.598 & 196.97 & 0.0753 \\
\hline & $\theta=0.1127$ & 0.1622 & & & & & \\
\hline Modified Weibull & $\delta=0.4049$ & 0.2011 & 95.380 & 196.761 & 197.114 & 199.31 & 0.0959 \\
\hline & $\alpha=1.6347$ & 0.2787 & & & & & \\
\hline Exponential & $\theta=0.5655$ & 0.06666 & 113.037 & 228.074 & 228.131 & 230.35 & 0.2806 \\
\hline
\end{tabular}
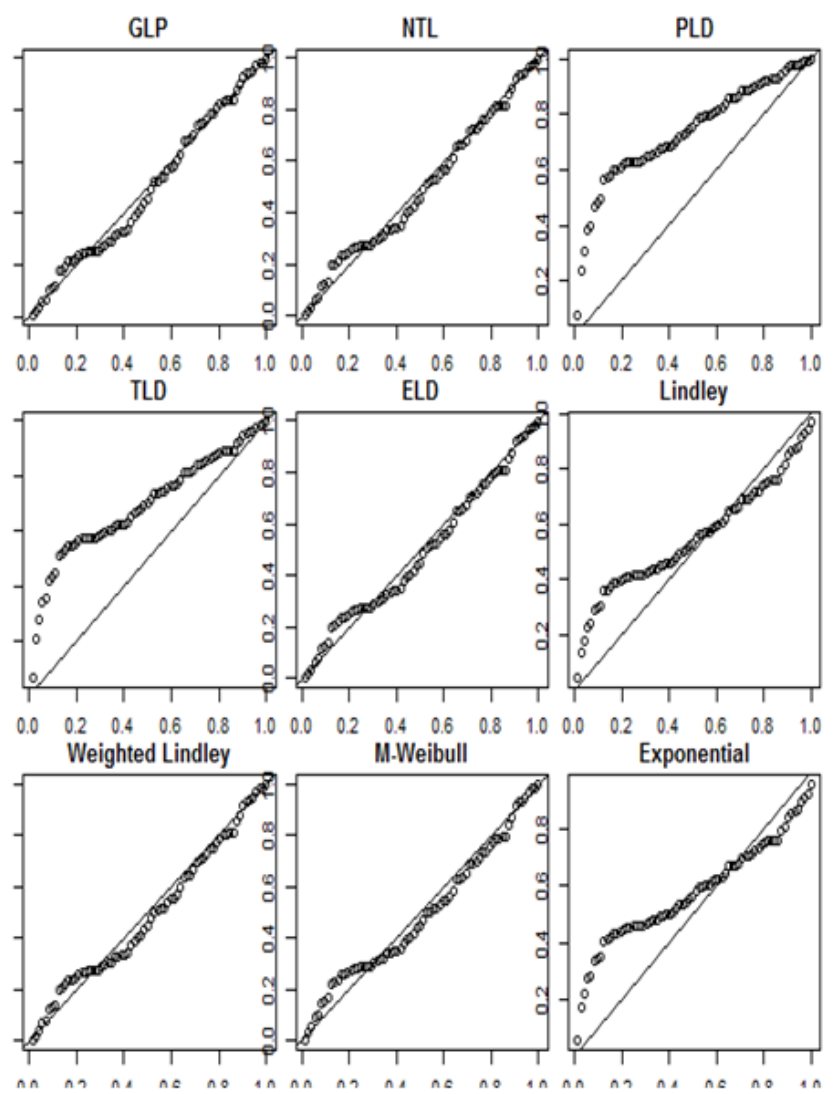

Figure 6: Probability plots for the fits GLP, NTL, PLD, TLD, ELD, LD, WL, MW and Exponential distributions of data set 2.

As we can see from Tables 1 and 2, our model with smallest values of AIC, AICC, BIC and K-S test statistic best fits the data. Figures 3 and 5 shows the empirical distribution compared to the rival models 
and 8 compares the fitted densities against the data, Figures 4 and 6 show the probability-probability plots, all confirm our findings of Tables 1 and 2.

\section{Generalizations}

In this section, we present generalizations of the GLP1 distribution.

In this section, we provide a general treatment by taking the probability density function of the cumulative distribution function of failure times to be given by $f(x)$ and $F(x)$, respectively. In this case, (1.8) generalizes to

$$
G(x)=\frac{(1-\pi)[1-\exp (-\lambda F(x))]}{1-e^{-\lambda}-\pi[1-\exp (-\lambda F(x))]},
$$

for $x>0, \lambda>0$, and $0<\pi<1$. The corresponding probability density function, hazard rate function and reversed failure rate function are

$$
\begin{aligned}
& g(x)=\frac{\lambda\left(1-e^{-\lambda}\right)(1-\pi) f(x)[\exp (-\lambda F(x))]}{\left[1-e^{-\lambda}-\pi[1-\exp (-\lambda F(x))]\right]^{2}}, \\
& h(x)=\frac{g(x)}{\bar{G}(x)}=\frac{\lambda\left(1-e^{-\lambda}\right)(1-\pi) f(x)[\exp (-\lambda F(x))]}{[\exp (-\lambda F(x))-\exp (-\lambda)]\left[1-e^{-\lambda}-\pi[1-\exp (-\lambda F(x))]\right]},
\end{aligned}
$$

and

$$
\operatorname{rh}(x)=\frac{g(x)}{G(x)}=\frac{\lambda\left(1-e^{-\lambda}\right) f(x)[\exp (-\lambda F(x))]}{[1-\exp (-\lambda F(x))]\left[1-e^{-\lambda}-\pi[1-\exp (-\lambda F(x))]\right]} .
$$

The shapes of (9.2) and (9.3) can be studied by taking their derivatives. Note that

$$
\frac{\partial \log g(x)}{\partial x}=\frac{\grave{f}(x)}{f(x)}-\lambda f(x)+\frac{2 \lambda \pi f(x)[\exp (-\lambda F(x))]}{1-e^{-\lambda}-\pi[1-\exp (-\lambda F(x))]},
$$

and

$$
\frac{\partial \log h(x)}{\partial x}=\frac{\partial \log g(x)}{\partial x}+\frac{\lambda f(x)[\exp (-\lambda F(x))]}{\exp (-\lambda F(x))-\exp (-\lambda)}-\frac{\lambda \pi f(x)[\exp (-\lambda F(x))]}{1-e^{-\lambda}-\pi[1-\exp (-\lambda F(x))]} .
$$

The quantile function corresponding to (9.1) is

$$
\mathrm{G}^{-1}(\mathrm{u})=\mathrm{F}^{-1}\left[\frac{-1}{\lambda} \log \left\{1-\frac{\mathrm{u}\left(1-\mathrm{e}^{-\lambda}\right)}{1-\pi+\mathrm{u} \pi}\right\}\right] .
$$

Using the series expansion, we can express (9.1) and (9.2) as mixtures, we can rewrite $G(x)$ as

$$
G(x)=\frac{(1-\pi)}{\left(1-e^{-\lambda}-\pi\right)} \sum_{k=0}^{\infty}\left(\begin{array}{c}
-1 \\
k
\end{array}\right)\left[\frac{\pi}{1-e^{-\lambda}-\pi}\right]^{k}[\exp (-k \lambda F(x))-\exp (-(k+1) \lambda F(x))],
$$

and

$$
g(x)=\frac{\lambda\left(1-e^{-\lambda}\right)(1-\pi)}{\left(1-e^{-\lambda}-\pi\right)^{2}} \sum_{k=0}^{\infty}\left(\begin{array}{c}
-1 \\
k
\end{array}\right)\left[\frac{\pi}{1-e^{-\lambda}-\pi}\right]^{k} f(x) \exp (-(k+1) \lambda F(x)) .
$$

\section{Concluding remarks}

There has been a great interest among statisticians and applied researchers in constructing flexible lifetime models to facilitate better modelling of survival data. Consequently, a significant progress has been made towards the generalization of some well-known lifetime models and their successful application to 
problems in several areas. In this paper, we introduce a new three-parameter distribution. We refer to the new model as the GLP distribution and study some of its mathematical and statistical properties. We provide the pdf, the cdf and the hazard rate function of the new model, explicit expressions for the moments. The model parameters are estimated by maximum likelihood. The new model is compared with nested and non-nested models and provides consistently better fit than other classical lifetime models. We hope that the proposed distribution will serve as an alternative model to other models available in the literature for modelling positive real data in many areas such as engineering, survival analysis, hydrology, and economics.

\section{References}

[1] H. S. Bakouch, B. M. Al-Zahrani, A. A. Al-Shomrani, V. A. A. Marchi, F. Louzada, An extended Lindley distribution, J. Korean Statist. Soc., 41 (2012), 75-85. 1.2

[2] T. Bjerkedal, Acquisition of Resistance in Guinea Pies infected with Different Doses of Virulent Tubercle, American J. Hygiene, 72 (1960), 130-148. 8.2

[3] M. E. Ghitany, D. K. Al-Mutairi, S. Nadarajah, Zero-truncated Poisson-Lindley distribution and its Applications, Math. Comput. Simulation, 79 (2008), 279-287. 1.2

[4] W. H. Gui, S. L. Zhang, X. M. Lu, The Lindley-Poisson distribution in lifetime analysis and its properties, Hacet. J. Math. Stat., 43 (2014), 1063-1077. 1.1

[5] E. Mahmoudi, H. Zakerzadeh, Generalized poisson-lindley distribution, Comm. Statist. Theory Methods, 39 (2010), 1785-1798. 1.2

[6] F. Merovci, Transmuted lindley distribution, Int. J. Open Prob. Comput. Sci. Math., 6 (2013), 63-72. 8.1

[7] S. Nadarajah, H. S. Bakouch, R. Tahmasbi, A generalized Lindley distribution, Sankhya B, 73 (2011), 331-359. 1.2

[8] S. Nadarajah, V. G. Cancho, E. M. M. Ortega, The geometric exponential Poisson distribution, Stat. Methods Appl., 22 (2013), 355-380. 1, 1.1

[9] A. Rényi, On measures of entropy and information, Proc. 4th Berkeley Sympos. Math. Statist. and Prob. (Univ. California Press, Berkeley), 1961 (1961), 547-561. 6.2

[10] M. Sankaran, The discrete Poisson-Lindley distribution, Biometrics, 26 (1970), 145-149. 1.2

[11] R. Shanker, S. Sharma, R. Shanker, A two-parameter Lindley distribution for modeling waiting and survival times data, Appl. Math., 4 (2013), 363-368. 1.2

[12] C. E. Shannon, A Mathematical Theory of Communication, Bell System Tech. J., 27 (1948), 379-423, 623-656. 6, 6.1

[13] H. Zakerzadah, A. Dolati, Generalized Lindley distribution, J. Math. Ext., 3 (2010), 13-25. 1.2

[14] H. Zakerzadeh, E. Mahmoudi, A new two parameter lifetime distribution: model and properties, arXiv preprint, 2012 (2012), 19 pages. 1.1 